\title{
Structural and sequence diversity of the transposon Galileo in the Drosophila willistoni genome
}

\author{
Juliana W Gonçalves ${ }^{1}$, Victor Hugo Valiati ${ }^{2 \dagger}$, Alejandra Delprat ${ }^{3}$, Vera L S Valente ${ }^{1^{* \dagger}}$ and Alfredo Ruiz ${ }^{3 \dagger}$
}

\begin{abstract}
Background: Galileo is one of three members of the $P$ superfamily of DNA transposons. It was originally discovered in Drosophila buzzatii, in which three segregating chromosomal inversions were shown to have been generated by ectopic recombination between Galileo copies. Subsequently, Galileo was identified in six of 12 sequenced Drosophila genomes, indicating its widespread distribution within this genus. Galileo is strikingly abundant in Drosophila willistoni, a neotropical species that is highly polymorphic for chromosomal inversions, suggesting a role for this transposon in the evolution of its genome.
\end{abstract}

Results: We carried out a detailed characterization of all Galileo copies present in the D. willistoni genome. A total of 191 copies, including 133 with two terminal inverted repeats (TIRs), were classified according to structure in six groups. The TIRs exhibited remarkable variation in their length and structure compared to the most complete copy. Three copies showed extended TIRs due to internal tandem repeats, the insertion of other transposable elements (TEs), or the incorporation of non-TIR sequences into the TIRs. Phylogenetic analyses of the transposase (TPase)-encoding and TIR segments yielded two divergent clades, which we termed Galileo subfamilies V and W. Target-site duplications (TSDs) in D. willistoni Galileo copies were 7- or 8-bp in length, with the consensus sequence GTATTAC. Analysis of the region around the TSDs revealed a target site motif (TSM) with a 15-bp palindrome that may give rise to a stem-loop secondary structure.

Conclusions: There is a remarkable abundance and diversity of Galileo copies in the D. willistoni genome, although no functional copies were found. The TIRs in particular have a dynamic structure and extend in different ways, but their ends (required for transposition) are more conserved than the rest of the element. The $D$. willistoni genome harbors two Galileo subfamilies ( $V$ and $W$ ) that diverged $\sim 9$ million years ago and may have descended from an ancestral element in the genome. Galileo shows a significant insertion preference for a 15-bp palindromic TSM.

Keywords: Transposable element, D. willistoni, Terminal inverted repeats, P superfamily, Target site duplications

\section{Background}

Transposable elements (TEs) are part of the middle repetitive portion of DNA that is able to move and replicate within the genome. They comprise a considerable fraction of many eukaryotic genomes and their sequences exhibit broad structural diversity. The wide range of transposition strategies adopted by TEs involve

\footnotetext{
* Correspondence: vera.valente@pq.cnpq.br

${ }^{\dagger}$ Equal contributors

'Programa de Pós-Graduação em Genética e Biologia Molecular, Departamento de Genética, Universidade Federal do Rio Grande do Sul (UFRGS), CP 15053, Porto Alegre, Rio Grande do Sul 91501-970, Brazil Full list of author information is available at the end of the article
}

either RNA (class 1 or retrotransposons) or DNA (class 2 or DNA transposons) intermediates. Selfish and thus in many respects indistinguishable in their behavior from parasites, these mobile genetic units increase in number within the genome because their rates of transposition are higher than those of spontaneous deletion. This evolutionary success of TEs is a major force shaping the genes and genomes of almost all organisms [1,2].

The movement and accumulation of TEs serves as a rich source of genetic material, with a strong impact on the evolutionary reorganization of the genomes of their bearers. However, it is now clear that inactive TEs also play a significant role in macroevolution, because the 
most influential contributions can arise and persist long after transposition activity has ceased, such that they are manifested as TE by-products. The selfish and parasitic characteristics of TEs ensure their long-standing residence within the host genome and imply their intimate co-evolutionary relationship with it [3].

The specific features of DNA transposons compared to other TEs enhance their influence in shaping eukaryotic genomes, including the capacity to excise imprecisely, jump locally, cause multiple double-strand breaks, and undergo alternative transposition [2]. The transposon Galileo was originally discovered in Drosophila buzzatii, in wich three segregating chromosomal inversions were shown to have been generated by ectopic recombination between Galileo copies [4-6]. Although Galileo has long terminal inverted repeats (TIRs) similar to those of Foldback-like elements, it is classified as a member of the $P$ superfamily of DNA transposons (class II, subclass 1, TIR elements order) based on the sequence of its putative transposase (TPase). Subsequently, Galileo was identified in six of the 12 sequenced Drosophila genomes of the two subgenera of Sophophora and Drosophila, indicating its widespread distribution within this genus. Although potentially active Galileo copies have not been found, non-autonomous copies are abundant in all species investigated [7]. In addition, two or more Galileo subfamilies coexisting within the same genome have been found in several cases: three subfamilies are present in D. buzzatii (G, K, and $\mathrm{N}$ for Galileo, Kepler and Newton), two in D. virilis (A and $\mathrm{B}$ ), and five in $D$. mojavensis (C, D, E, F, and $\mathrm{X})[6-8]$.

According to in silico predictions, Galileo is strikingly abundant in Drosophila willistoni [7], the most widespread neotropical species of the genus Drosophila $[9,10]$, with an extensive gene arrangement polymorphism on all chromosomes [11-20]. This high intraspecific polymorphism for chromosomal inversions and Galileo abundance suggest a role for Galileo in the generation of inversions in $D$. willistoni and related species. We have an ongoing project to test this hypothesis by identifying and isolating the breakpoints of $D$. willistoni natural polymorphic inversions. As a first step in this in this project, we carried out an exhaustive search for and characterization of the Galileo copies present in the $D$. willistoni genome. A careful and detailed annotation of 191 Galileo sequences revealed that they vary considerably in length and structure, ranging from nearly-complete to containing only one TIR. Two Galileo subfamilies with a substantial nucleotide divergence were found by phylogenetic analysis of TPase-encoding and TIR segments. In addition, by analyzing the preferred target sequence of Galileo in D. willistoni, we identified a palindromic target site motif (TSM).

\section{Results}

\section{Characterization of Galileo copies in the D. willistoni genome}

We characterized 191 Galileo copies in the D. willistoni genome (details are given in Additional file 1), classifying them into six groups according to their structure (Table 1 and Figure 1): (A) nearly-complete; (B) two TIRs and a partial TPase-encoding segment; $(\mathrm{C})$ one TIR and a partial TPase-encoding segment; (D) two TIRs; (E) one TIR only; and (F) a TPase-encoding segment. Only one nearlycomplete copy, containing two TIRs and a nearly-complete TPase-encoding segment, was found. This copy, identified in previous work (GenBank: BK006360.1) [7], is 4386-bp long and harbors a long ORF (coordinates 984-3698) encoding a 905-amino-acid TPase. The only mismatch is in the start codon, with ACG = Thr instead of the canonical ATG = Met; thus, this copy cannot be functional. Nonetheless, this putative TPase is similar in size and composition to other Galileo elements [7]. Protein functional analysis, performed using InterProScan 4 [21], revealed the presence of a THAP domain (PF05485) in residues 14-93 (2E-12) and a THAP-domain containing a protein 9 domain (PTHR10725) in residues 251-884 (1E-61). THAP is a DNA-binding domain present in TPases of the $P$ superfamily; this domain includes a $\mathrm{Zn}$-coordinating $\mathrm{C} 2 \mathrm{CH}$ signature and four other invariant residues (P, W, F, and $\mathrm{P}$ ) that are also required for DNA binding [8]. These eight residues are fully conserved at positions $\mathrm{C} 16, \mathrm{C} 21, \mathrm{P} 40$, W49, C67, H70, F71, and P87 of the putative Galileo TPase. The second conserved domain included the triad

Table 1 Galileo copies characterized in the Drosophila willistoni genome

\begin{tabular}{ccccc}
\hline Group & Number of copies & Copies with inserted TE & Copies with flanking TE & Copies with inserted and flanking TE \\
\hline A & 1 & 0 & 1 & 0 \\
B & 7 & 11 & 0 & 0 \\
C & 26 & 11 & 19 & 3 \\
D & 124 & 0 & 1 & 2 \\
E & 2 & 6 & 7 & 1 \\
F & 31 & 33 & 28 & 3 \\
Total & 191 & & & 9 \\
\hline
\end{tabular}

$\mathrm{TE}$, transposable element. 




Figure 1 Galileo copies identified in the Drosophila willistoni genome were classified into the following six groups according to structure: A) Nearly-complete with two terminal inverted repeats (TIRs) and nearly-complete transposase (TPase)-encoding segment (GenBank: BK006360.1); B) two TIRs and a TPase segment; C) one TIR and a TPase segment; D) two TIRs; E) one TIR; and F) a TPase segment. The black arrows represent the TIRs. The blue middle region in A represents the nearly-complete TPase-encoding segment. The green middle region ( $\mathbf{B}, \mathbf{C}$, and $\mathbf{F}$ ) represents a partial TPase-encoding segment. The black lines in D indicate the spacing sequences between the $5^{\prime}$ and $3^{\prime}$ TIRs. These sequences do not show homology at the nucleotide level to any known sequence in the databases.

$\mathrm{DDE}$ and the motif $\mathrm{D}(2) \mathrm{H}$, which is present in the catalytic domain of cut-and-paste TPases of the P superfamily [22] at positions D327, D415, E642, and D449(2)H452.

Most ( 89\%) of the copies with preserved terminal sequences are flanked by identical target-site duplications (TSDs). Approximately 17.3\% (33 copies) contain other elements inserted within them, $14.7 \%$ (28 copies) have other elements inserted at the Galileo termini, and 4.7\% (9 copies) have both inserted and flanking elements (Table 1). In one case, we identified a full-length $P$ element (99\% identity with the D. melanogaster P element) [23], possibly with imperfect TSDs (CGCTAGCC/GGCTA GCG) inserted within the Galileo copy, that contained only fragments of TIRs and identical TSDs. Of the copies with a TPase-encoding segment only (group F), 58\% (18 copies) are located at the ends of short scaffolds ( $\leq 5,598$-bp); thus, they may be incomplete, either because the rest of the sequence is present somewhere else or it is missing. None of the copies in groups B-F have an intact ORF encoding a putatively functional TPase (i.e., all characterized copies are non-autonomous; with variable portions of the TPase-coding region).

\section{TIR structural variation}

Galileo copies in the D. willistoni genome exhibit remarkable structural variation. In particular, the TIRs vary considerably in length and structure compared to the TIRs of the nearly-complete copy (Figure 1), which are 765/757-bp long and have 99\% identity (omitting indels). The 3' TIR has a 69-bp overlap with the TPase-coding segment (Figure 1). Thus, the final piece of this segment is repeated (in reverse orientation) at the $5^{\prime}$ TIR. This is a unique trait among the described Galileo transposons [7]. Also, there are two AT-rich segments, with the 136-bp segment located in the 5' TIR (coordinates 528663) and the 137-bp segment located in the $3^{\prime}$ TIR (coordinates 3732-3868).

Three Galileo copies were found to display significantly extended TIRs and each one is flanked by identical TSDs (Figure 2). The longest copy (112) is 9021-bp long, including TIRs of 4246-bp and 4680-bp (5' and 3', respectively; see Additional file 1). This copy contains only two TIRs and it lacks a TPase-coding segment. However, the TIRs are notable for their striking length and repetitive structure (Figure 3). They contain direct tandem repeats and an insertion of another TE in addition to the AT-rich segments. This longest copy is the only one with direct tandem repeats within the TIRs. The repeats are $\sim 140$-bp long and located approximately 1710-bp and 1730-bp from the $5^{\prime}$ and $3^{\prime}$ ends, respectively. The $5^{\prime}$ TIR contains three repeat regions, two that are 275-bp long (2 tandem repeats) and another that is 443-bp long (3.2 repeats). On the 3 ' end, we annotated two longer repeat regions, a 995-bp region (6.8 repeats) and a 1246-bp region (9 repeats) (Figures 2 and 3). The TIRs of this copy contain fragments of two additional transposons: P element and Mar. At the $5^{\prime}$ and $3^{\prime}$ ends, we identified one fragment (36-bp) of a $P$ element (86.1\% identity with the D. bifasciata P element, according to the database; coordinates: 2900-2935) [24]. Of the seven fragments of Mar that were annotated, two are 107-bp long (one at each end) and the other five are 


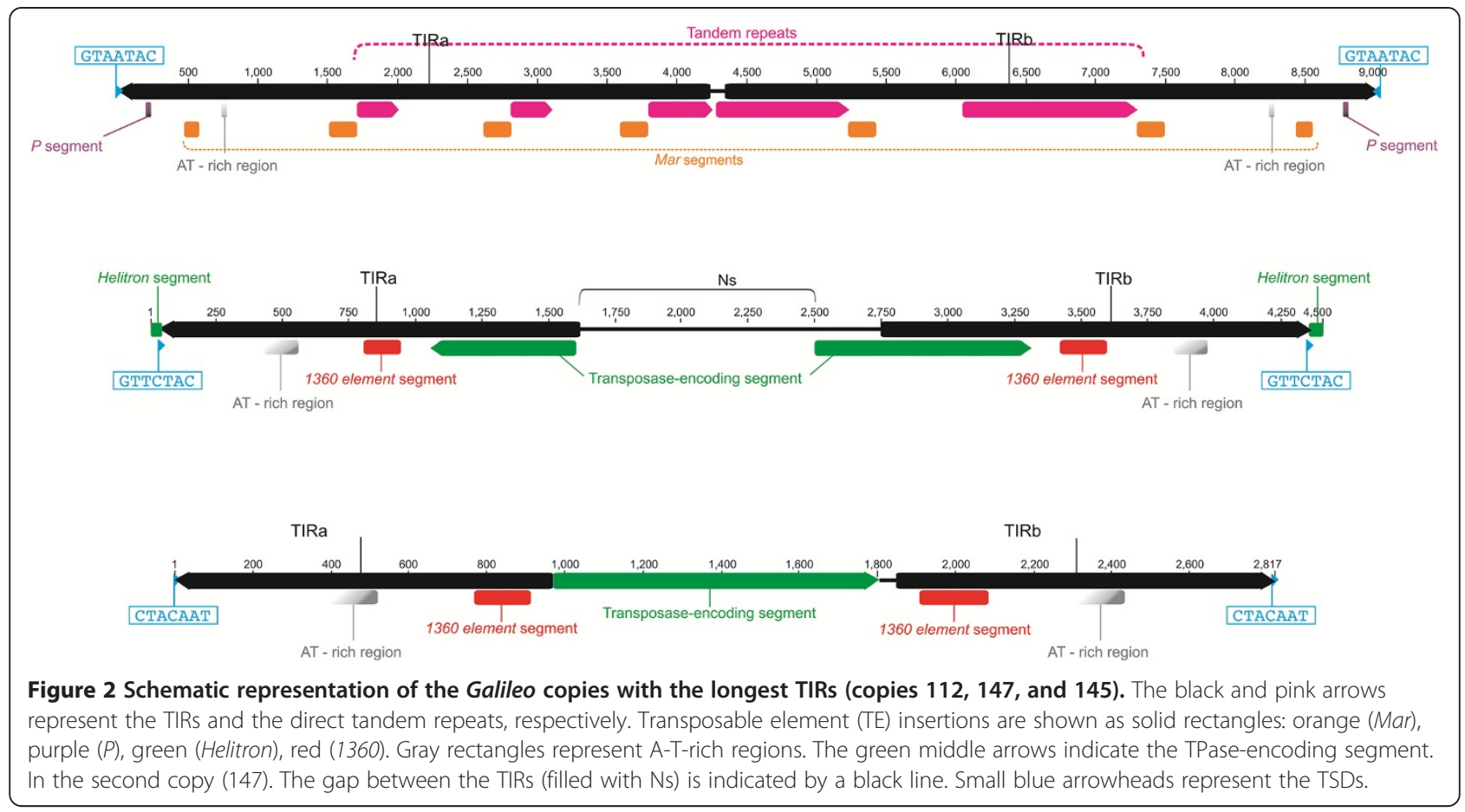

202-bp long (98.1\% and $94.5 \%$ identity with D. willistoni Mar, respectively; coordinates 491-597 and 299-500) [25].

The copy (147) with the second longest TIRs (1575-bp and 1608-bp; $97.5 \%$ identical) is 4306-bp long (Figure 2). The TIRs are composed of: 130-bp of AT-rich sequence, 140-bp and 172-bp stretches ( $5^{\prime}$ and $3^{\prime}$ TIR, respectively) similar to transposon 1360 (also known as Hoppel or ProtoP element; $85.7 \%$ identity with coordinates 4020-3869 and 84.9\% identity with coordinates 38694079, respectively) [26,27], and at least 545-bp of the Galileo TPase (coordinates: 3700-3162). Thus, in this
Galileo copy, TPase-encoding segments are repeated, forming part of the TIRs (Figure 2). In addition, there is a 872-bp gap between the TIRs (filled with Ns) that may hide a larger TPase fragment. The third copy (145) has TIRs that are 959-bp (99.9\% identical) in length, with 132-bp of AT-rich sequence and the same fragments of the1360 transposon present in the copy previously described. These last two copies are similar in their structure and have $99.8 \%$ identity over the first 959-bp. However, they have different TSDs, indicating that they are independent insertions.



Figure 3 Dot-matrix plot of the longest Galileo copy in the $D$. willistoni genome. The copy structure contains two long TIRs. The principal diagonal line with disruptions (insertions and deletions) shows the alignment between the two TIRs. Additional repetitive regions are represented by diagonally striped rectangles. 
Galileo sequence diversity in the $D$. willistoni genome

We aligned the TPase-coding segments from 26 Galileo copies and used a 488 -bp region (coordinates: 26993131) to build a phylogenetic tree using maximum-likelihood (ML) and Bayesian inference (BI) methods. The phylogenetic trees of the TPase-coding sequences created using the two methods were similar and recovered the same two clades with significant statistical support (Figure 4). The two clades showed a substantial nucleotide divergence between them $(20 \%-50.3 \%)$ and were termed Galileo subfamilies V and W. The analysis placed the nearly-complete copy within the $\mathrm{W}$ subfamily. Assuming a Drosophila synonymous substitution rate of 0.016 substitutions per nucleotide/myr [28], we estimated the split between the two subfamilies to $\sim 9$ million years ago (Mya). The two subfamilies have a modest mean divergence between copies within each one (4.7 and 6.7\%, respectively) compared with the mean divergence between them (24\%) (Table 2).

We also aligned the TIR regions and built a phylogenetic tree with 238 homologous segments (the first 100-bp). The topologies of the TIR trees also yielded two clades, with 11\%-26.6\% nucleotide divergence (Figure 5). Several copies $(72,114,115,116,151)$, in addition to the nearly-complete copy (166), contain both the initial portion of the TIR and a TPase-encoding fragment. The phylogenetic placement of these copies suggests that the two clades in the TIR phylogeny correspond to the above-defined $\mathrm{V}$ and $\mathrm{W}$ subfamilies (Figure 5 and Additional file 2). Subfamily V was represented by copies with extended TIRs (145 and 147) that have the homologous TIR region (the first 100-bp). There was no significant difference in the lengths of the TIRs among subfamilies. The mean divergence between copies of the two subfamilies was $13.6 \%$, whereas the mean divergence within subfamilies was much smaller (1.3\% and $3.1 \%)$ (Table 3). The divergence within subfamilies includes the estimates between copies and between the two TIRs within copies.

The consensus sequences for the terminal 40-bp segment in Galileo subfamilies V and W differed by 4 bp (10\%). A comparison of the 40 terminal bp region conserved in 14 Galileo sequences of diverse species and subfamilies showed a total of 17 conserved nucleotides (Figure 6).

\section{Target site duplication and target site motif}

In most $D$. willistoni Galileo copies, the TSDs were 7-bp in length, as similarly reported in D. buzzatti [29]. However, we identified three copies $(89,127$, and 179) in which the TSDs were 8-bp long (see Additional file 1). Comparison of the 118 flanking sequences of those Galileo copies with the 7-bp TSD suggested that the consensus sequence of their preferential insertion site is GTATTAC (Figure 7).

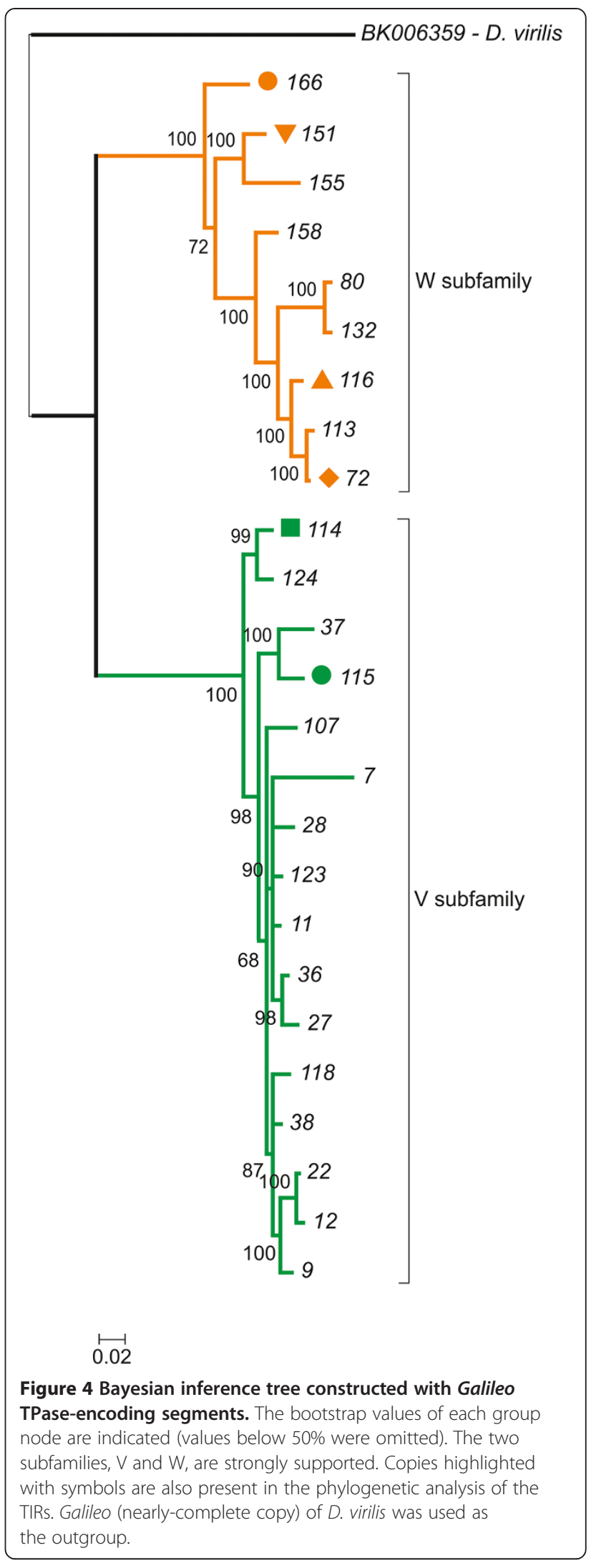


Table 2 Nucleotide divergence estimates (\%) for the Galileo subfamilies using transposase-coding sequences

\begin{tabular}{lccc}
\hline & V subfamily & W subfamily & Galileo of $\boldsymbol{D}$. virilis \\
\hline V subfamily & $4.7 \pm 0.60$ & & \\
W subfamily & $24.02 \pm 2.19$ & $6.66 \pm 0.086$ & \\
Galileo of D. virilis & $32.34 \pm 2.52$ & $30.08 \pm 2.60$ & - \\
\hline
\end{tabular}

Galileo (nearly-complete copy) of $D$. virilis was used.

The majority-rule consensus sequence for the flanking sequences of $D$. willistoni Galileo copies suggested that the insertion sites are localized at the center of the AT-rich region. Analysis of the 93-bp surrounding the TSDs revealed a target site motif (TSM) with a 15-bp palindromic pattern composed of 7-bp duplicated upon insertion plus 4-bp on either side of Galileo (Figure 7).

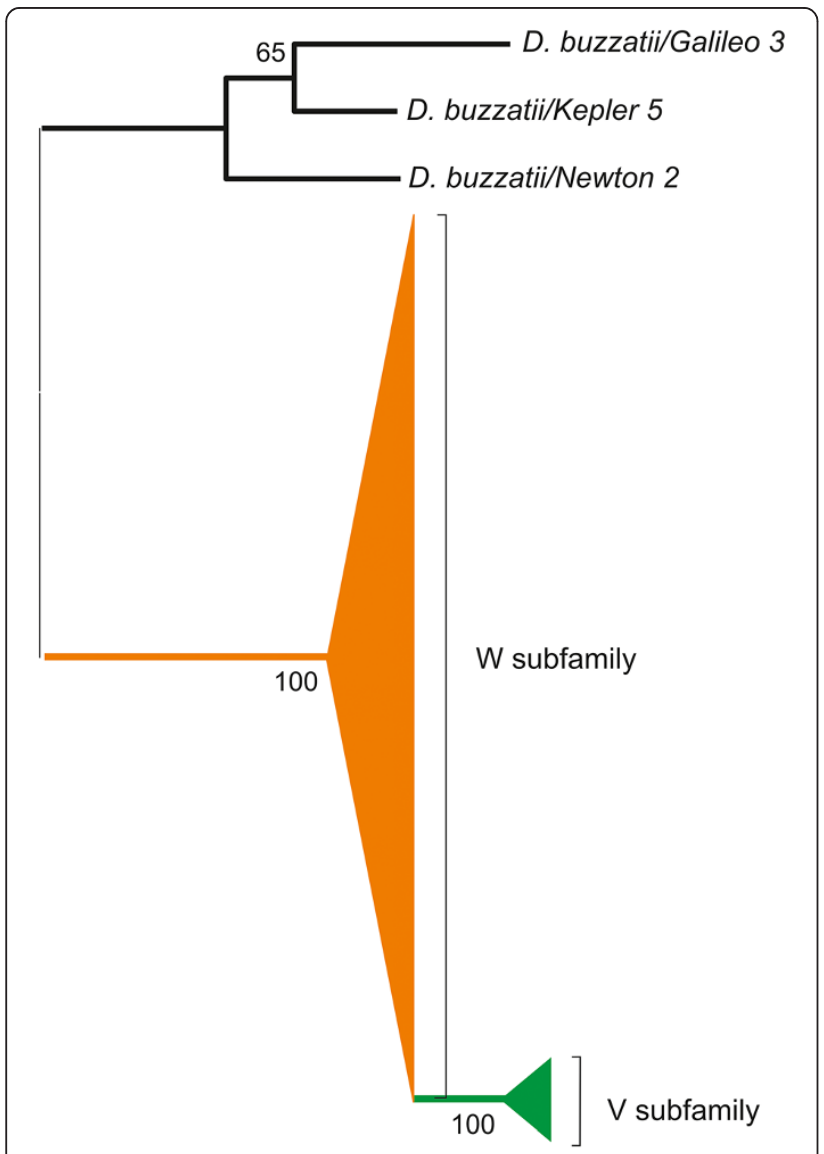

$0 . \mathrm{H}^{0}$

Figure 5 Bayesian inference tree showing the relationships among Galileo TIRs. The groups were compressed to facilitate visualization; therefore, 114 and 24 sequences were omitted from the $W$ and $V$ subfamilies, respectively. The bootstrap values of each group node are indicated (values below $50 \%$ were omitted). The two subfamilies, V and W, are strongly supported. The Galileo, Kepler, and Newton subfamilies of D. buzzatii were used as the outgroup in the phylogenetic analysis.
Two pentanucleotides, AANGT and ACNTT, were identified on the $5^{\prime}$ and $3^{\prime}$ halves of the TSM [30]. This motif could adopt a stem-loop secondary structure when denatured.

\section{Discussion}

In the present study, we used different search strategies and a detailed manual annotation to fully characterize Galileo copies in the Drosophila willistoni genome. In contrast to previous work [7], which reported on 28 copies, this study presents information on 191 copies. The long term goal of this project is to contrast the hypothesis that Galileo generated some of the D. willistoni chromosomal inversions segregating in natural populations. The detailed annotation of all Galileo copies present in the $D$. willistoni genome will greatly assist in the interpretation of the breakpoint sequences.

\section{Galileo structural variation}

Putatively functional copies of Galileo were not found, although one nearly complete copy harbors an ORF coding for a 905-amino-acid TPase (after curating a mismatch in the start codon). Among the non-autonomous copies with TPase segments, the majority ( 63.6\%) were composed of TIRs and a spacing region. In addition, they exhibit a remarkable structural variation, particularly in the TIRs. Galileo, along with two other transposons, P-element and 1360, are members of the $P$ superfamily [31]. $P$ elements move to a new site through a non-replicative process, i.e., the cut-and-paste mechanism of transposition, in which the excised copy leaves behind a double-strand gap [32]. Because gap repair is not always efficient, whether via homologous recombination or using the sister chromatid strand as a template, defective copies are often generated due to abortion, slippage, or template switching in the course of transposition and repair [2,33]. Furthermore, because transposons are dispersed repeats in the genome, non-allelic homologous recombination or ectopic recombination events are likely, thereby increasing the probability of exchange between two copies and affecting the structure of the sequences. These molecular processes can explain the gradient of Galileo copies found in the D. willistoni genome, ranging from an almost-complete copy to defective copies restricted to the TIRs, with various degrees of degeneration.

Moreover, Galileo displays dynamic restructuring. A recent analysis of the Drosophila mojavensis genome [8] identified two patterns of extension for Galileo TIRs: (1) expansion of the direct tandem repeats and (2) recruitment of internal sequences (non-TIR segments) into the TIRs. In the $D$. willistoni genome, we identified direct tandem repeats within the TIRs, but in a single copy only (the longest one, Figure 2). We also found evidence of recruitment of non-TIR segments into the TIRs. 
Table 3 Nucleotide divergence estimates (\%) for the Galileo subfamilies using the terminal inverted repeat regions

\begin{tabular}{lccc}
\hline & V subfamily & W subfamily & Subfamilies of $\boldsymbol{D}$. buzzatii \\
\hline V subfamily & $3.08 \pm 0.09$ & & \\
W subfamily & $13.61 \pm 3.57$ & $1.31 \pm 0.024$ & $28.41 \pm 4.02$ \\
Subfamilies of D. buzzatii & $52.92 \pm 3.83$ & $48.51 \pm 4.91$ & \\
\hline
\end{tabular}

Galileo subfamilies K, G and N of D. buzzatii were used.

Remarkably, in the nearly-complete copy, a 69-bp final piece of the ORF is now part of the $3^{\prime}$ 'TIR, and it is repeated (in reverse orientation) at the end of the $5^{\prime}$ 'TIR. In other copies, the overlap between the ORF and TIRs is even greater. For instance, in copy 147, the segment of the ORF recruited to the TIRs is more than 500-bp long (Figure 2). This is, so far, a unique trait of Galileo transposons [7]. Finally, we found a third pattern of TIR extension: the insertion of another TE into one of the TIRs, which eventually may be transferred to the other TIR, ultimately becoming a part of both. The TE fragments are not occasional insertions in one Galileo TIR; rather they are part of the $5^{\prime}$ and $3^{\prime}$ ends in transposing copies. We detected the insertion of three elements in the longest TIRs, i.e., P, Mar, and 1360. The first two were previously studied in $D$. willistoni [34-36], but the origin of the 1360 fragment is obscure because in D. willistoni transposon 1360 is missing [7].

\section{Two Galileo subfamilies in the $D$. willistoni genome}

In previous work [7], a limited number of Galileo copies were isolated from the $D$. willistoni genome, and a subsequent phylogenetic analysis did not detect a significant structure. Here, our phylogenetic analysis, based on an increased number of copies, revealed two strongly supported clades, which we named subfamilies $\mathrm{V}$ and $\mathrm{W}$. The two clades were evident in phylogenetic analyses carried out using either a segment of the ORF or the final 100-bp of the TIRs. Although in our study only six copies were shared between the two phylogenies, two in subfamily V $(114,115)$ and four in subfamily W $(72,116$, 151 and 166), the results are congruent and suggest the same grouping in the two subfamilies. The presence of Galileo subfamilies within the same genome seems to be the rule rather than the exception, as was previously found in D. buzzatii (subfamilies G, K, and N), D. virilis (subfamilies A and B), and D. mojavensis (subfamilies C, D, E, F, and X) [6-8]. Furthermore, the coexistence of different subfamilies, subgroups, or variants of TEs was reported in studies of Bari [37] and Gypsy [38] in Drosophila, $P$ in Anopheles gambiae and Drosophila [39,40], and mariner in insects and humans [41,42], among others.

How have these Galileo copies differentiated in the genome of D. willistoni? Horizontal transfer (HT) and vertical diversification are the two main hypotheses that explain the coexistence of different subfamilies in the same genome [2]. HT would account for the appearance of the two subfamilies, via two independent events of Galileo invasion in the D. willistoni genome. Several mechanisms and vectors have been proposed to explain HT events. In Drosophila parasites and parasitoids, such as mites and wasps, intracellular symbiotic bacteria, such as Wolbachia and spiroplasms, are possible vectors of TEs [43]. HT can also result from an introgression, as reported in the willistoni subgroup [44-46], and is a potential mechanism for $P$ element spreading among this subgroup [47]. Although the HT hypothesis in the case of Galileo has yet to be disproven, our data suggest that, based on the landscape of this transposon in D. willistoni, the copies instead diverged from an ancestral element in



Figure 6 Comparison of the TIRs ends. A consensus sequence was constructed for the $\mathrm{V}$ and W subfamilies of the Galileo TIRs in D. willistoni. Alignments of the 40-bp TIRs of each Galileo subfamily and species are shown. Identical positions (17) in all sequences are marked in black, and the $80 \%$ and $60 \%$ conserved positions in green and gray, respectively. 


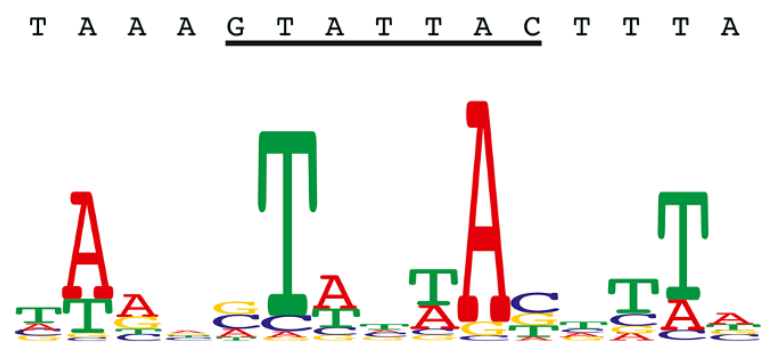

Figure 7 Sequence logo and consensus sequence for the 7-bp TSD and the 15-bp target site motif. The sequence underlined in black is the target preference for Galileo in the D. willistoni genome.

the genome. Although no complete copies of Galileo have been found, its functional differentiation would have had to be driven by specific selective pressures, resulting in the formation of two distinct Galileo TPases to overcome cellular repression of transposition. We identified Galileo copies composed of TIRs with conserved TPase site affinity in the genome; these could have served as a source for the other defective copies. Furthermore, HT and vertical diversification are not mutually exclusive; thus, successive invasions and structural variations may have occurred during the diversification of TEs. Concerning the preservation of Galileo TIRs, the mean divergence for these sequences was only one half $(\sim 13.6 \%)$ of that for the TPase-encoding segment ( $24 \%)$. Under a neutral evolution model, the same degree of divergence would be expected; however, in the case of Galileo, there are more constraints in the terminal segment (100-bp) of its TIRs than in its TPase-encoding segment because the former are required for transposition.

\section{Galileo insertional preference}

DNA transposons generate, upon insertion, direct duplications of short genome sequences (TSDs). In D. buzzatii, a comparison of the 19 flanking sequences suggested that Galileo generates 7-bp TSDs with the consensus sequence GTAGTAC [48]. A larger sample (106 Galileo copies) in six sequenced Drosophila genomes (D. ananassae, D. pseudoobscura, D. persimilis, D. willistoni, D. virilis, and D. mojavensis) identified the consensus sequence GTANTAC [7]. We found that the Galileo TSDs in D. willistoni are typically 7 -bp but, as occurs in most $P$ element insertions, three Galileo copies had TSDs of 8-bp. Additionally, by comparing 118 Galileo copies flanked by identical 7-bp sequences, we were able to infer that the preferential insertion site has a consensus sequence of GTATTAC, in which the fourth position differs from that occurring in Galileo copies in D. buzzatii. These findings are in agreement with those of a study of six Drosophila genomes. Linheiro and Bergman [30] measured the degree of target specificity for different elements in D. melanogaster. They found that 1360 and $P$ elements seem to have a relatively low degree of target specificity. Galileo seems to have a higher target specificity than either 1360 or the $P$ element. Accordingly, it can be detected with a lower number of insertions [7,29,48].

A previous study identified a 14-bp palindromic pattern centered on the 8 -bp TSD generated by $P$ element insertion [49]. Sequence motifs at TE target sites are always palindromes that extend beyond the TSD [30]. Here, by analyzing the region around the TSDs, a 15-bp palindrome was identified; in addition, the Galileo TSM also had a general tendency to be AT-rich. Although the tendency in the TSMs of both $P$ element and 1360 is to have an ANAGT motif in the $5^{\prime}$ half and an ACTNT motif in the 3' half, the Galileo TSM while palindromic, is not identical in sequence (AANGT and ACNTT, respectively).

\section{Conclusions}

Our detailed analysis of 191 Galileo copies revealed an enormous variety in their size and structure. In some copies, there were different forms of TIR extension, including internal duplications, recruitment of the final piece of the TPase-encoding ORF into the TIRs and secondary TE insertions in one TIR that subsequently become part of both TIRs. Two Galileo subfamilies (termed $\mathrm{V}$ and $\mathrm{W}$ ) coexist in the D. willistoni genome. They are evident in the phylogenetic trees of both the TPase-encoding and the TIR segments. However, phylogenetic analysis showed that the divergence between and within subfamilies is smaller in the TIR segment than in the TPase-encoding segment, presumably because the former is required for transposition. Galileo shows a stronger target preference that 1360 or P-element, the other two members of the $P$ superfamily.

\section{Methods}

\section{Bioinformatic searches}

The D. willistoni genome sequence was used for in silico analyses. Candidate Galileo elements were identified by querying the nearly-complete copy of Galileo detected in the D. willistoni genome in previous work [7], terminal inverted repeats (TIRs), and segments of the transposase (TPase)-encoding ORF isolated by experimental searches of Galileo in D. willistoni (Gonçalves et al. in preparation). Blast searches of the Drosophila willistoni genome were performed using FlyBase [50], with default parameters and without a low complexity filter, to identify copies with simple and complex repeats. The applied threshold of scores had an e-value of $<10^{-4}$. To accept a search hit, we compared previously characterized copies and identified characteristic structures.

When a segment of the Galileo TPase was used as the query subject, to identify TIRs and target site duplications (TSDs), pairwise comparisons of upstream and 
downstream flanking sequences (up to 5000-bp, if available) were carried out. TSDs were identified by aligning 50-bp upstream and downstream of the TIRs of the TEs. The target site motif (TSM) was constructed by concatenating the flanking sequence upstream of the element insertion, containing the TSD (50-bp), and the flanking sequence (43-bp) downstream from the element insertion, lacking both this element and the TSD. Hits were considered part of the same Galileo copy if arranged in the proper orientation at a distance of $<5 \mathrm{~Kb}$.

\section{Annotation of the Galileo copies}

The detected sequences were manually annotated using different tools, most of which were implemented using Geneious R6, created by Biomatters [51], and custom Blast searches using specific Galileo and Drosophila TE databases. To avoid and discard false automatic identifications, all hits from each search were manually curated. Similarity searches were used to identify and annotate the insertions of other TEs inside Galileo by Blast searches, carried out using the National Center for Biotechnology Information (NCBI) [52] and RepeatMasker [53] databases. Default parameters were applied, except for basic options, which we set as follows: cross-match in the search engine, slow in speed/sensitivity, and specify Drosophila in DNA source. To verify the presence of direct repeats, we used the Tandem Repeat Finder program, with default parameters [54]. Thus, we identified the following regions in each Galileo copy: TIRs, the TPasecoding region, and insertions and repeats.

\section{Phylogenetic analysis}

Phylogenetic trees were built using the TPase-coding sequences ( 630-bp) and the homologous TIR region (100-bp). The sequences were aligned with MAFT software [55]. Phylogenetic analyses were conducted with the maximum likelihood (ML) method, using PHYML 2.4.4 [56] and Bayesian inference of phylogeny (BI) using MrBayes 3.1.2 [57], applying default priors and three heated, one cold Markov chains and running each analysis from two random starting points. For TPase segments, the Akaike's information criterion (AIC, Akaike 1974) indicated that the $\mathrm{HKY}+\mathrm{G}$ model [58] was the best fit-model of sequence evolution $(-\ln L=2502.9929$, $\mathrm{AIC}=5015.9858)$ and of the gamma-distribution shape parameter (5.7262). The Markov chain Monte Carlo search was run with 10,000,000 generations (repeated two times), with sampling conducted every 1,000 generations. The first $25 \%$ of the trees were discarded as "burn-in", at which time the chain reached stationarity, ensuring that the average split frequencies between the runs was $<1 \%$. For the TIR sequences, the AIC indicated that the GTR model [59] with an equal gamma distribution rate was the best fit-model of sequence evolution
$(-\operatorname{lnL}=785.0532, \quad$ AIC $=1586.1063)$. The Markov chain Monte Carlo search was run with 10,000,000 generations (repeated tree times) and sampled every 1,000 generations. The first $25 \%$ of the trees were discarded as "burn-in", at which time the chain reached stationarity. MEGA version 5.1 was used to calculate the average divergence within and between Galileo subfamilies, and the p-distance model and 1,000 bootstrap replications were used to date the divergence between the V and $\mathrm{W}$ subfamilies, calibrating the tree with the synonymous substitution rate of 0.016 substitutions per site per million years, as calculated for Drosophila genes with a low codon usage bias $[28,60]$.

\section{Identification of insertion sites}

Insertion sites were analyzed by extracting the flanking sequences (50-bp) upstream and downstream of the element insertion, i.e., those lacking the element and TSD. For this analysis, we restricted the data to those from insertions for which the TSD sequence from each end of the element could be independently determined. To examine the potential secondary structure formed at the insertion site, we used the m-fold web server [61] to analyze the majority rule consensus sequence of the sequences around the TSDs; default parameters were applied, except in the case of the "folding temperature", which was set to $23^{\circ} \mathrm{C}$.

\section{Additional files}

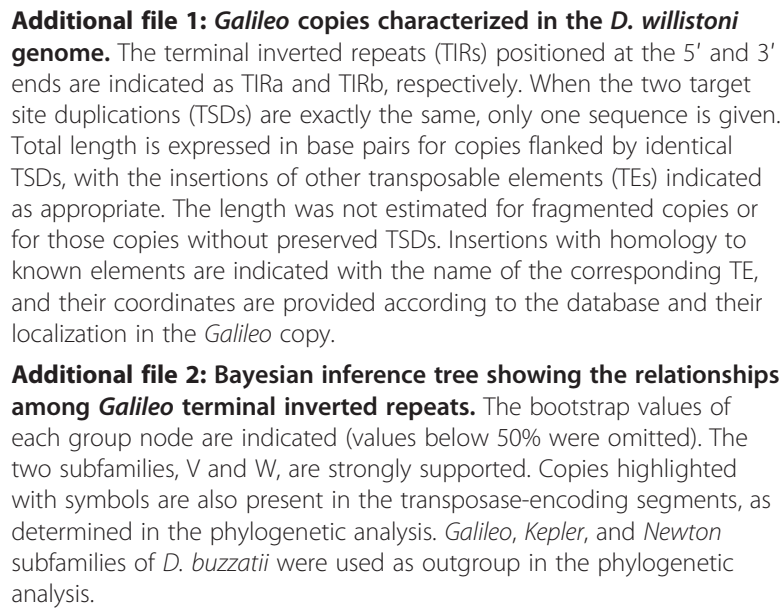

Additional file 2: Bayesian inference tree showing the relationships among Galileo terminal inverted repeats. The bootstrap values of each group node are indicated (values below 50\% were omitted). The two subfamilies, V and W, are strongly supported. Copies highlighted with symbols are also present in the transposase-encoding segments, as determined in the phylogenetic analysis. Galileo, Kepler, and Newton subfamilies of $D$. buzzatii were used as outgroup in the phylogenetic analysis.

Competing interests

The authors declare that they have no competing interests.

\section{Authors' contributions}

JWG carried out the in silico searches, the results analysis, and wrote the manuscript. VHV, AD, VLSV, and AR assisted with the analysis and manuscript writing. AR and VLSV provided funding and facilities for the study. AR conceived and coordinated the study. All authors read and approved the final manuscript. 


\section{Acknowledgements}

This study was supported by research grants and fellowships from Brazilian CNPq (Conselho Nacional de Desenvolvimento Científico e Tecnológico), PRONEX-FAPERGS (Programa de Apoio aos Núcleos de Excelência - Fundação de Amparo à Pesquisa do Estado do Rio Grande do Sul - 10/0028-7), and CAPES (Coordenação de Aperfeiçoamento de Pessoal de Nível Superior). Additional funds were provided by grant BFU2011-30476 from Ministerio de Ciencia e Innovación (Spain).

\section{Author details}

'Programa de Pós-Graduação em Genética e Biologia Molecular, Departamento de Genética, Universidade Federal do Rio Grande do Sul (UFRGS), CP 15053, Porto Alegre, Rio Grande do Sul 91501-970, Brazil. ${ }^{2}$ Programa de Pós-Graduação em Biologia: Diversidade e Manejo de Vida Silvestre, Universidade do Vale do Rio dos Sinos (UNISINOS), CP 275, São Leopoldo, Rio Grande do Sul 93022-000, Brazil. ${ }^{3}$ Departament de Genètica i de Microbiologia, Universitat Autònoma de Barcelona (UAB), 08193 Bellaterra, Barcelona, Spain.

Received: 26 April 2014 Accepted: 9 September 2014

Published: 13 September 2014

\section{References}

1. Brookfield JFY: The ecology of the genome - mobile DNA elements and their hosts. Nat Rev Genet 2005, 6:128-136.

2. Feschotte C, Pritham EJ: DNA transposons and the evolution of eukaryotic genomes. Annu Rev Genet 2007, 41:331-368.

3. Feschotte $C$ : The contribution of transposable elements to the evolution of regulatory networks. Nat Rev Genet 2008, 9:397-405

4. Cáceres M, Ranz JM, Barbadilla A, Long M, Ruiz A: Generation of a Widespread Drosophila Inversion by a Transposable Element. Science 1999, 285:415-418.

5. Casals F, Cáceres M, Ruiz A: The Foldback-like transposon Galileo is involved in the generation of two different natural chromosomal inversions of Drosophila buzzatii. Mol Bio Evol 2003, 20:674-685.

6. Delprat A, Negre B, Puig M, Ruiz A: The transposon Galileo generates natural chromosomal inversions in Drosophila by ectopic recombination. PLoS One 2009, 4:13.

7. Marzo M, Puig M, Ruiz A: The Foldback-like element Galileo belongs to the $P$ superfamily of DNA transposons and is widespread within the Drosophila genus. PNAS 2008, 105:2957-2962.

8. Marzo M, Bello X, Puig M, Maside X, Ruiz A: Striking structural dynamism and nucleotide sequence variation of the transposon Galileo in the genome of Drosophila mojavensis. Mob DNA 2013, 4:1.

9. Spassky B, Richmond RC, Perez Salas S, Pavlovsky O, Mourão CA, Hunter AS, Hoenigsberg H, Dobzhansky T, Ayala FJ: Geography of the sibling species related to Drosophila willistoni, and of the semispecies of the Drosophila paulistorum complex. Evolution 1971, 25:129-143.

10. Dobzhansky T, Powell JR: The Willistoni Group of Sibling Species of Drosophila. In Handbook of Genetics. Edited by King RC. New York: Plenum Press; 1975:589-622.

11. Da Cunha AB, Burla H, Dobzhansky T: Adaptative chromosomal polymorphism in Drosophila willistoni. Evolution 1950, 4:212-235.

12. Da Cunha AB, Dobzhanky T, Pavlovsky O, Spassky B: Genetics on natural populations. XXVIII. Suplementary data on the chromosomal polymorphism in Drosophila willistoni in its relation to the environment. Evolution 1959, 13:389-404

13. Da Cunha AB, Dobzhansky T: A further study of chromosomal polymorphism in Drosophila willistoni in the relation to the environment. Evolution 1954, 8:119-134.

14. Valente VLS, Morales NB: New inversions and qualitative description of inversion heterozygotes in natural populations of Drosophila willistoni. Rev Bras Genet 1985, 8:167-173.

15. Valente VLS, Araújo AM: Chromosomal polymorphism, climatic factors and variation in population size of Drosophila willistoni. Heredity 1986, 57:149-160.

16. Valente VLS, Ruszczyk A, Santos RA: Chromosomal polymorphism in urban Drosophila willistoni. Rev Bras Genet 1993, 16:307-319.

17. Valente VLS, Rohde C, Valiati VH, Morales NB, Goñi B: Chromosomal inversions occurring in Uruguayan populations of Drosophila willistoni. Dros Inf Serv 2001, 84:55-59.
18. Valente VLS, Goñi B, Valiati VH, Rohde C, Morales NB: Chromosomal polymorphism in Drosophila willistoni populations from Uruguay. Genet Mol Bio 2003, 26:163-173.

19. Rohde C, Cristina A, Garcia L, Valiati VH, Valente VLS: Chromosomal evolution of sibling species of the Drosophila willistoni group. I. Chromosomal arm IIR (Muller's element B ). Genetica 2006, 126:77-88.

20. Bhutkar A, Schaeffer SW, Russo SM, Xu M, Smith TF, Gelbart WM: Chromosomal rearrangement inferred from comparisons of 12 Drosophila genomes. Genetics 2008, 179:1657-1680.

21. Quevillon E, Silventoinen V, Pillai S, Harte N, Mulder N, Apweiler R, Lopez R. InterProScan: protein domains identifier. Nucleic Acids Res 2005, 33:116-120

22. Yuan Y-W, Wessler SR: The catalytic of all eukaryotic cut-and-paste transposase superfamilies. Proc Natl Acad Sci U S A 2011, 108:1-6.

23. O'Hare K, Rubin GM: Structures of $P$ transposable elements and their sites of insertion and excision in the Drosophila melanogaster genome. Cell 1983, 34:25-35.

24. Hagemann S, Miller JW, Pinsker W: Identification of a complete P-element in the genome of Drosophila bifasciata. Nucleic Acids Res 1992, 20:409-413.

25. Holyoake AJ, Kidwell MG: Vege and Mar: two novel hAT MITE families from Drosophila willistoni. Mol Biol Evol 2003, 20:163-167.

26. Kapitonov W, Jurka J: Molecular paleontology of transposable elements in the Drosophila melanogaster genome. Proc Natl Acad Sci 2003, 100:6569-6574.

27. Reiss D, Quesneville H, Nouaud D, Andrieu O, Anxolabehere D: Hoppel, a $P$-like element without introns: a $P$-element ancestral structure or a retrotranscription derivative? Mol Biol Evol 2003, 20:869-879.

28. Sharp PM, Li WH: On the rate of DNA sequences evolution in Drosophila. J Mol Evol 1989, 28:398-402.

29. Cáceres M, Puig M, Ruiz A: Molecular characterization of two natural hotspots in the Drosophila buzzatii genome induced by transposon insertions. Genome Res 2001, 11:1353-1364.

30. Linheiro RS, Bergman CM: Whole genome resequencing reveals natural target site preferences of transposable elements in Drosophila melanogaster. PLoS One 2012, 7:e30008.

31. Rius N, Delprat A, Ruiz A: A divergent P-element and its associated MITE, BuT5, generate chromosomal inversions and are widespread within the Drosophila repleta species group. Genome Biol Evol 2013, 5:1127-1141.

32. Engels WR, Johnson-Schlitz DM, Eggleston WB, Sved J: High- frequency $P$ element loss in Drosophila is homolog dependent. Cell 1990, 62:515-525.

33. Brunet F, Giraud T, Godin F, Capy P: Do deletions of Mos7-like elements occur randomly in the Drosophilidae family? J Mol Evol 2002, 54:227-234.

34. Daniels SB, Peterson KR, Strausbaugh LD, Kidwell MG, Chovnick A: Evidence for horizontal transmission of the $P$ transposable element between Drosophila species. Genetics 1990, 124:339-355.

35. Regner LP, Pereira MS, Alonso CE, Abdelhay E, Valente VL: Genomic distribution of $P$ elements in Drosophila willistoni and a search for their relationship with chromosomal inversions. J Hered 1996, 87:191-198.

36. Deprá M, Ludwig A, Valente VLS, Loreto ELS: Mar, a MITE family of hAT transposons in Drosophila. Mob DNA 2012, 3:13

37. Moschetti R, Chlamydas S, Marsano RM, Caizzi R: Conserved motifs and dynamic aspects of the terminal inverted repeat organization within Bari-like transposons. Mol Genet Genomics 2008, 279:451-461.

38. Ludwig A, Valente VLS, Loreto ELS: Multiple invasions of Errantivirus in the genus Drosophila. Insect Mol Biol 2008, 17:113-124.

39. Quesneville H, Nouaud D, Anxolabehere D: $P$ elements and MITE relatives in the whole genome sequence of Anopheles gambiae. BMC Genomics 2006, 7:214.

40. Loreto ELS, Zambra FMB, Ortiz MF, Robe L: New Drosophila P-like elements and reclassification of Drosophila P-elements subfamilies. Mol Genet Genomics 2012, 287:531-540.

41. Robertson HM, MacLeod EG: Five major subfamilies of mariner transposable elements in insects, including the Mediterranean fruit fly, and related arthropods. Insect Mol Biol 1993, 2:125-139.

42. Robertson HM, Martos R: Molecular evolution of the second ancient human mariner transposon, Hsmar2, illustrates patterns of neutral evolution in the human genome lineage. Gene 1997, 205:219-228.

43. Loreto ELS, Carareto CM, Capy P: Revisiting horizontal transfer of transposable elements in Drosophila. Heredity 2008, 100:545-554

44. Ehrman L, Powell JR: The Drosophila Willistoni Species Group. In The Genetics and Biology of Drosophila. Edited by Ashburner M, Carson HL, Thompson JN. New York: Academic Press; 1982:193-225. 
45. Cordeiro AR, Winge H: Levels of Evolutionary Divergence of Drosophila Willistoni Sibling Species. In Genetics of Natural Populations: The Continuing Importance of Theodosius Dobzhansky. Edited by Levine L. New York: Columbia University Press; 1995:262-280.

46. Robe L, Cordeiro J, Loreto ELS, Valente VLS: Taxonomic boundaries, phylogenetic relationships and biogeography of the Drosophila willistoni subgroup (Diptera : Drosophilidae). Genetica 2010, 138:601-617.

47. Silva JC, Kidwell MG: Horizontal transfer and selection in the evolution of P elements. Mol Bio Evol 2000, 17:1542-1557.

48. Casals F, Cáceres M, Manfrin MH, González J, Ruiz A: Molecular characterization and chromosomal distribution of Galileo, Kepler and Newton, three foldback transposable elements of the Drosophila buzzatii species complex. Genetics 2005, 169:2047-2059.

49. Liao GC, Rehm EJ, Rubin GM: Insertion site preferences of the $P$ transposable element in Drosophila melanogaster. PNAS 2000, 97:3347-3351.

50. A database of drosophila genes \& genomes. http://flybase.org/blast.

51. Geneious. http://www.geneious.com.

52. Basic local alignment search tool. http://blast.ncbi.n/m.nih.gov/Blast.cgi.

53. RepeatMasker. http://www.repeatmasker.org.

54. Benson G: Tandem repeats finder: a program to analyze DNA sequences. Nucleic Acids Res 1999, 27:573-580.

55. Katoh K, Misawa K, Kuma K, Miyata T: MAFFT: a novel method for rapid multiple sequence alignment based on fast Fourier transform. Nucleic Acids Res 2002, 30:3059-3066

56. Guindon S, Gascuel O: A simple, fast, and accurate algorithm to estimate large phylogenies by maximum likelihood. Syst Biol 2003, 52:696-704.

57. Ronquist F, Huelsenbeck JP: MRBAYES 3: Bayesian phylogenetic inference under mixed models. Bioinformatics 2003, 19:1572-1574.

58. Hasegawa M, Kishino H, Yano T: Dating of the human-ape splitting by a molecular clock of mitochondrial DNA. J Mol Evol 1985, 22:160-174.

59. Rodríguez FJ, Oliver JL, Marín A, Medina JR: The general stochastic model of nucleotide substitution. J Theor Biol 1990, 142:485-501.

60. Tamura K, Peterson D, Peterson N, Stecher G, Nei M, Kumar S: MEGA5: molecular evolutionary genetics analysis using maximum likelihood, evolutionary distance, and maximum parsimony methods. Mol Biol Evol 2011, 28:2731-2739.

61. The mfold Web server. http://www.bioinfo.rpi.edu/applications/mfold.

doi:10.1186/1471-2164-15-792

Cite this article as: Gonçalves et al:: Structural and sequence diversity of the transposon Galileo in the Drosophila willistoni genome. BMC Genomics 2014 15:792.

\section{Submit your next manuscript to BioMed Central and take full advantage of:}

- Convenient online submission

- Thorough peer review

- No space constraints or color figure charges

- Immediate publication on acceptance

- Inclusion in PubMed, CAS, Scopus and Google Scholar

- Research which is freely available for redistribution 